\title{
A Model for Phase Transitions Under Dynamic Compression
}

\author{
C. W. Greeff ${ }^{1}$
}

Received: 14 September 2016/ Accepted: 1 October 2016/Published online: 13 October 2016

(C) Society for Experimental Mechanics, Inc 2016

\begin{abstract}
An approach is described for simulating phase transitions among an arbitrary number of phases under dynamic compression. The time evolution of the phase fractions $\lambda_{i}$ is governed by the master equation, which naturally incorporates the constraints $\sum_{i} \lambda_{i}=1$ and $0 \leq \lambda_{i} \leq 1$. The rates are taken to be strongly nonlinear functions of the thermodynamic driving forces. The implementation of the method is described, and illustrative applications to phase transitions in $\mathrm{Zr}$ are shown. The relation of the model to irreversible thermodynamics is discussed. A modification for the case where domain growth limits the transformation rate is described. This variant gives sigmoidal transformation-time curves, with the second phase fraction proportional to $t^{3}$ at early time.
\end{abstract}

\section{Introduction}

Dynamic compression experiments have played an important role in the investigation of material properties at high pressures, and in particular high pressure phase transitions [10]. These studies have benefited from comparisons of continuum level simulations with time-resolved wave profile data $[5,9,13,24]$. Such comparisons allow the data to be correlated with equation of state (EOS) and phase diagram information from a variety of sources, and permit inferences to be made about transformation kinetics at time scales that range typically from a few to hundreds of ns.

\footnotetext{
C. W. Greeff

greeff@lanl.gov

1 Los Alamos National Laboratory, Los Alamos, New Mexico 87545, USA
}

The models of Andrews [2] and Hayes [16] have been influential on subsequent simulations of this type. These models treat the equation of state of the phase transforming material as that of a mixture, whose component phases are assumed to be in pressure and temperature equilibrium. The mole fractions of the phases, here denoted by $\left\{\lambda_{i}\right\}$, are dynamical variables, which evolve in time towards the equilibrium state. The model discussed here is of this general type, but with some significant differences. The equation of motion for the phase fractions is the master equation, which, builds in both the constraints that $\sum_{i} \lambda_{i}=$ 1 and $0 \leq \lambda_{i} \leq 1$. Under a fixed pressure and temperature, the Andrews-Hayes formulation gives $\lambda_{i}$ that vary linearly in time until they reach one or zero, at which time the transformation rate is set to zero. Time evolution under the master equation leads to $\lambda_{i}$ approaching zero or one asymptotically. This is more consistent with experimental data $[18,26]$ from static compression experiments, and leads to better agreement with dynamic data $[9,13,24]$.

The other main difference between the present model and the Andrews-Hayes model concerns the dependence of the rate on the thermodynamic driving force. Whereas in Refs. $[2,16]$ the transformation rate depends linearly on the thermodynamic driving force, $G_{j}-G_{i}$, the present model has a strongly nonlinear dependence. This was originally found to be necessary to describe dynamic data on the $\alpha-\omega$ transition in Ti and Zr. For these, the shock induced transition occurs well above the equilibrium transition pressure, but occurs rapidly enough to be observable. This requires a rate that is very low, just above the equilibrium boundary, and increases very rapidly. This picture is reinforced by observations of on $\mathrm{Ti}$ [26] under static compression, which show an exponential dependence of the transformation rate on the pressure. The model has also 
been found to be useful for the $\beta-\gamma$ transition in Sn [9], and gives good results in unpublished calculations on melting of Sn after shock and release [22].

An earlier version of the model presented here has been briefly described in other publications [13, 15, 24]. These earlier works focused on the case of two phases. This paper describes the more recent version of the model, which is oriented towards handling an arbitrary number of phases, and brings in the master equation as the time evolution equation. Section 2 of this paper gives a detailed presentation of the model, with a discussion of the relation to irreversible thermodynamics. Section 3 describes the current numerical implementation. Illustrative applications are shown in Sect. 4, and an extension of the model for the case where the rate is limited by domain growth is presented in Sect. 5. This extension brings in an explicit dependence of the transformation rate on the phase fractions, as well as the thermodynamic state, and leads to an early time behavior $\lambda \propto t^{3}$ for the second phase fraction. Finally there is a summary and outlook.

\section{Model}

The model operates at a macroscopic level, where individual phase domains are not resolved. Instead, the mole fractions of the various phases, denoted $\left\{\lambda_{i}\right\}$ characterize the state. A multi-phase EOS consisting of Helmholtz free energies $F_{i}\left(V_{i}, T\right)$ for the various phases is required. It is assumed that the significant domains are large enough to be characterized by their bulk free energies. Pressure and temperature equilibrium are imposed, allowing the temperature and individual phase specific volumes $\left\{V_{i}\right\}$ to be determined given the specific internal energy $E$ and the total specific volume $V=\sum_{i} \lambda_{i} V_{i}$. The kinetic model determines the evolution of the phase fractions $\left\{\lambda_{i}\right\}$. From the point of view of a hydrodynamic simulation, the model acts as an EOS with a time-evolving internal state.

In the two-phase version of the model, the time evolution of the second phase fraction was written $[13,15,24]$

$\dot{\lambda_{2}}=\left(1-\lambda_{2}\right) R=\lambda_{1} R$

where the rate $R$ is a function of the thermodynamic state. Making the rate of change proportional to $\lambda_{1}=1-\lambda_{2}$ leads to asymptotic approach to completion. Equation (1) was used under circumstances where the rate of the reverse $2 \rightarrow 1$ transition was negligible. Allowing for reverse transformations, and generalizing to arbitrary number of phases leads to the master equation,

$\dot{\lambda}_{i}=\sum_{j \neq i}\left(\lambda_{j} R_{j i}-\lambda_{i} R_{i j}\right)$

(Time derivatives refer to a fixed Lagrangian coordinate, or material point, throughout.) The $R_{i j}$ are non-negative, and give the rate of transformation from phase $i$ to phase $j$, relative to the fraction of phase $i$. They are functions of the thermodynamic driving force for the transformation, here taken to be the Gibbs free energy difference $G_{i}-G_{j}$. The factor $1-\lambda_{2}$ was included in Eq. (1) to improve agreement with shock wave profile data. In Avrami's formulation [3, 8], this factor enters as a geometrical correction for second phase domains growing into regions that have already transformed. This factor is automatically included within the master equation formulation, Eq. (2). It is straightforward to see that $\sum_{i} \dot{\lambda}_{i}=0$, preserving the normalization $\sum_{i} \lambda_{i}=1$. In addition, Eq. (2) enforces the constraints $0 \leq \lambda_{i} \leq 1$. For instance, suppose that $\lambda_{i}$ approaches 1 . Due to the normalization condition, the remaining $\lambda_{j \neq i}$ must approach zero, and the first term in Eq. 2, which is positive, approaches zero. The second term is negative or zero. Thus, $\lambda_{i}$ stops increasing as it approaches 1. Similar considerations apply as $\lambda_{i}$ approaches 0 . The result is that evolution by Eq. 2 allows the phase fractions to asymptotically approach the limiting values 0 and 1 , but never to exceed them.

The functional dependence of $R$ on $G_{i}-G_{j}$ introduced in Refs. $[13,15]$ is

$$
R_{i j}=\theta\left(G_{j}-G_{j}\right) \frac{v_{i j}}{B_{i j}}\left(G_{i}-G_{j}\right) \exp \left[\left(G_{i}-G_{j}\right)^{2} / B_{i j}^{2}\right]
$$

where $v_{i j}$ and $B_{i j}$ are material parameters for the $i \rightarrow j$ transition, and $\theta$ is the Heaviside step function. An implication of Eq. (3) is that there is an effective threshold driving force below which a phase transition is not observable. This is illustrated in Fig. 1. In a given experiment, there will some time scale $t_{\max }$ which is the longest time observable. For instance, in a shock experiment, this will be on the order of the shock transit time. Processes occurring slower than $R_{i j} \sim 1 / t_{\max }$ will be too slow to impact the observations. This leads the a threshold value of $G_{i}-G_{j}$ which is non-zero. This is shown in Fig. 1 as the intersection of the red dashed line with the solid black curve. The point $G_{i}-G_{j}=0$ is the equilibrium phase boundary. In order to drive the transition $i \rightarrow j$ at an observable rate, the driving force $G_{i}-G_{j}$ must exceed the threshold. Similar considerations apply to the reverse transition, meaning that the transition is effectively hysteretic on any finite time scale. Because of the exponential dependence in Eq. (3), the threshold value of $G_{i}-G_{j}$ is weakly dependent on the experimental conditions, such as sample thickness, that determine $t_{\max }$.

There is also a minimum observable transition time scale $t_{\min }$. This may be set by the experimental observation, or by other material response properties. For instance, in experiments on the $\alpha-\omega$ transition in $\mathrm{Zr}$ [24], the rise time of the plastic wave is on the order 


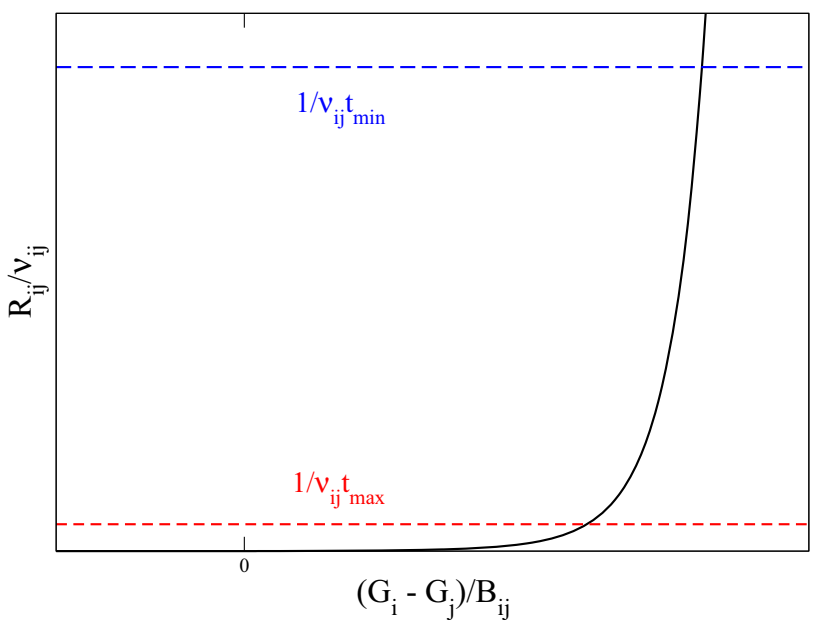

Fig. 1 (color online) Thresholding effect of the rate equation. The solid black curve is the rate $R_{i j}$ as a function of the driving force $G_{i}-G_{j}$ according to Eq. (3). The time scales $t_{\max }$ and $t_{\min }$ are the maximum and minimum observable. The intersection of the red dashed curve with the black curve illustrates the thresholding effect. The driving force must exceed the intersection point in order for the transformation to be observable. Above the intersection of the blue dashed curve with the black curve, the transformation is effectively instantaneous, and the functional form of $R_{i j}$ is irrelevant.

of $10 \mathrm{~s}$ of $\mathrm{ns}$, and is resolved by the VISAR measurements. Transitions occurring at rates much higher than $R_{i j}=1 / t_{\min }$ will be effectively instantaneous as far as can be discerned, and the functional form of $R\left(G_{i}-G_{j}\right)$ will cease to be relevant. An alternative form to Eq. (3) is discussed below in Sect. 4 that leads to essentially identical results.

The second law requires that the spontaneous evolution of a system with fixed volume and energy must lead to an increase of the entropy. Equivalently [23], for a system at fixed volume and temperature, the Helmholtz free energy must decrease. For the mixture of phases considered here, the total Helmholtz free energy is

$$
F(V, T)=\sum_{i} \lambda_{i} F_{i}\left(V_{i}, T\right)
$$

and its time derivative is

$$
\dot{F}=\sum_{i} \dot{\lambda}_{i} F_{i}+\lambda_{i} \frac{\partial F_{i}}{\partial V_{i}} \dot{V}_{i}
$$

Minimizing the total free energy with respect to the individual phase volumes, with the constraint $V=\sum_{i} \lambda_{i} V_{i}$ included through a Lagrange multiplier, results in equality of the individual phase pressures, $\frac{\partial F_{i}}{\partial V_{i}}=-P_{i}=-P$, where the common pressure, $P$, is the Lagrange multiplier. The second term in Eq. (5) becomes $-P \sum_{i} \lambda_{i} \dot{V}_{i}$. Fixed total volume leads to $\sum_{i} \lambda_{i} \dot{V}_{i}=-\sum_{i} \dot{\lambda}_{i} V_{i}$. Including these, Eq. (5) becomes
$\dot{F}=\sum_{i} \dot{\lambda}_{i} G_{i}$

Inserting Eq. (2) for $\dot{\lambda}_{i}$, and interchanging $i$ and $j$ in the second term, we have

$$
\dot{F}=-\sum_{i, j \neq i} \lambda_{j}\left(G_{j}-G_{i}\right) R_{j i}<0
$$

The inequality requires that $R_{j i}$ be non-zero only when the thermodynamic driving force $G_{j}-G_{i}$ is positive.

The result, Eq. (7), is not surprising, but the method for obtaining it is illuminating. The derivation shows how the condition of pressure equilibrium is consistent with the Gibbs free energy being the driving force. If the free energy were generalized from Eq. (4), for example, by including interfacial free energies, the condition of pressure equilibrium and the thermodynamic driving force would both be modified in order to maintain consistency with the second law. The Helmholtz formulation is useful for generalizations that include shear stress. In that case the condition of fixed volume, whose role is to impose no mechanical work by the environment, becomes the condition of no shape change of the overall volume element.

\section{Implementation}

The above model has been implemented into a simple onedimensional, planar continuum code (hydro code). Some details of this implementation are given here. The multiphase model interfaces to the code like a standard EOS. The specific volume $V=A / \rho$, where $A$ is the molar mass, and internal energy $E$ are given as inputs. The individual phase free energies $F_{i}\left(V_{i}, T\right)$ are given as analytic functions $[5,12,14]$ and the first and second derivatives of $F$ (e.g. $\partial P / \partial V)$ are evaluated along with $F$. The internal state of the model consists of the temperature $T$, phase fractions $\lambda_{i}$, individual phase volumes $V_{i}$. The individual phase Gibbs free energies $G_{i}$ are stored as well. At every time step, the time evolution equation, Eq. (2) is integrated. This is done by evaluating the functions $R_{i j}$ using the Gibbs free energies $G_{i}$ from the last time step, and updating the $\lambda_{i}$ with a forward Euler step. In order to maintain the constraint $0 \leq \lambda_{i} \leq 1$ with finite time step, the $R_{i j}$ are limited so that $R_{i j} \Delta t<r_{\max }$, where $r_{\max }<1$. Typically, I have taken $r_{\max }=$ 0.90 .

After updating the $\lambda_{i}$, the volumes $V_{i}$ and temperature $T$ are evaluated by solving the conditions of pressure equilibrium, $P_{i}\left(V_{i}, T\right)=P$ subject to the specified total volume $V=\sum_{i} \lambda_{i} V_{i}$ and internal energy $E=\sum_{i} \lambda_{i} E_{i}\left(V_{i}\right.$, $T)$. The volumes are updated by

$V_{i}^{n+1}=V_{i}^{n}+\Delta V_{i}$, 
where,

$\Delta V_{i}=\left(\bar{P}-P_{i}\right) / \frac{\partial P_{i}}{\partial V_{i}}$

Imposing the requirement,

$\sum_{i} \lambda_{i} V_{i}^{n+1}=V$

leads to

$$
\begin{aligned}
\bar{P}= & \left(V-\sum_{i} \lambda_{i} V_{i}+\sum_{i} \lambda_{i} P_{i} / \frac{\partial P_{i}}{\partial V_{i}}\right) / \\
& \left(\sum_{i} \lambda_{i} / \frac{\partial P_{i}}{\partial V_{i}}\right) .
\end{aligned}
$$

Similarly, the temperature is updated using,

$$
\Delta T=\left(E-\sum_{i} \lambda_{i} E_{i}\right) /\left(\sum_{i} \lambda_{i} \frac{\partial E_{i}}{\partial T}\right) .
$$

An iteration consists of one update of the volumes and one of the temperature. An error criterion is calculated at each iteration,

err $=\sum_{i} \frac{\left|\Delta V_{i}\right|}{V_{i}}+\frac{|\Delta T|}{T}$

and iterations are terminated when err is less than some tolerance, which has typically been set to $10^{-6}$. After convergence is reached, the pressure is returned, and the internal state is saved. The Gibbs free energies $G_{i}$ are used for the time evolution, and the volumes $V_{i}$ and temperature $T$ are used as the starting point for the iterations in the next step.

The time step is typically limited by stability of the iterations for equal pressure. In the current implementation, the stable time step is sufficiently small that there is no advantage to using a higher order method for the evolution of the phase fractions. Improvements to the robustness of the pressure equilibrium solution, which allow for larger time steps, would change this.

\section{Illustrations}

Here I show some illustrative applications of the model. For this, I use the EOS for Zr published in Ref. [12]. This EOS covers three solid phases: the ambient $\alpha$ phase, the high-pressure $\omega$, and the high-temperature and high-pressure $\beta$ phase [29]. Helmholtz free energies for each phase are given as functions of $V$ and $T$, in the widely used form of a sum of static lattice, nuclear motion and electronic excitation terms. The parameter values were determined from a combination of ambient pressure thermodynamic data, dynamic and static compression data, and first principles calculations.

The phase diagram for this EOS is shown in Fig. 2. An important feature of the EOS for the present work is that the equilibrium $\alpha-\omega$ phase boundary intersects the room temperature isotherm, Hugoniot, and principle isentrope at $P=2.3 \mathrm{GPa}$. The Hugoniot intersects the $\omega-\beta$ boundary at $24 \mathrm{GPa}$, while the principle isentrope crosses it at $30 \mathrm{GPa}$.

The first set of illustrative simulations consist of impacts of an Al flyer on $\mathrm{Zr}$, with a window of LiF. Figure 3 shows the simulated velocity at the $\mathrm{Zr} / \mathrm{LiF}$ interface, as would typically be observed in experiments. The Al flyer and the $\mathrm{Zr}$ sample are both $1 \mathrm{~mm}$ thick. The impactor velocity is varied from 0.5 to $3.0 \mathrm{~km} / \mathrm{s}$ in increments of $0.5 \mathrm{~km} / \mathrm{s}$. The corresponding shock stress in the $\mathrm{Zr}$ ranges from 5 to $38 \mathrm{GPa}$. The kinetic parameters are assumed to be symmetric, $B_{i j}=B_{j i}, v_{i j}=v_{j i}$, and are taken to be the same for all pairs of phases for this illustration. The values are $v=2.5 \times 10^{4} \mathrm{~s}^{-1}, \quad B=400 \mathrm{~J} / \mathrm{mol}$. These are realistic parameters for the $\alpha-\omega$ transition in high purity $\mathrm{Zr}$, and match experiments reported in [24] well. Shear stress is modeled with an elastic, perfectly plastic model with a shear modulus of $36 \mathrm{GPa}$, and a yield stress of $0.4 \mathrm{GPa}$. The simulations use 500 zones $2 \mu \mathrm{m}$ thick in the $\mathrm{Zr}$. The time step is $5 \times 10^{-5} \mu \mathrm{s}$, which corresponds to $c \Delta t / \Delta x \approx 0.1$. This time step is approximately $1 / 3$ the value required to maintain stability of the iterative solver described in Sect. 3.

A flyer velocity of $0.5 \mathrm{~km} / \mathrm{s}$ produces a shock pressure of $5 \mathrm{GPa}$, which is above the equilibrium $\alpha-\omega$ transition pressure, but due to the thresholding effect described in Fig. 1, there is very little transformation to the $\omega$ phase, with peak $\omega$ fraction less than $2 \%$. At $1.0 \mathrm{~km} / \mathrm{s}$, the $\alpha-\omega$ transformation goes to completion, but slowly enough to

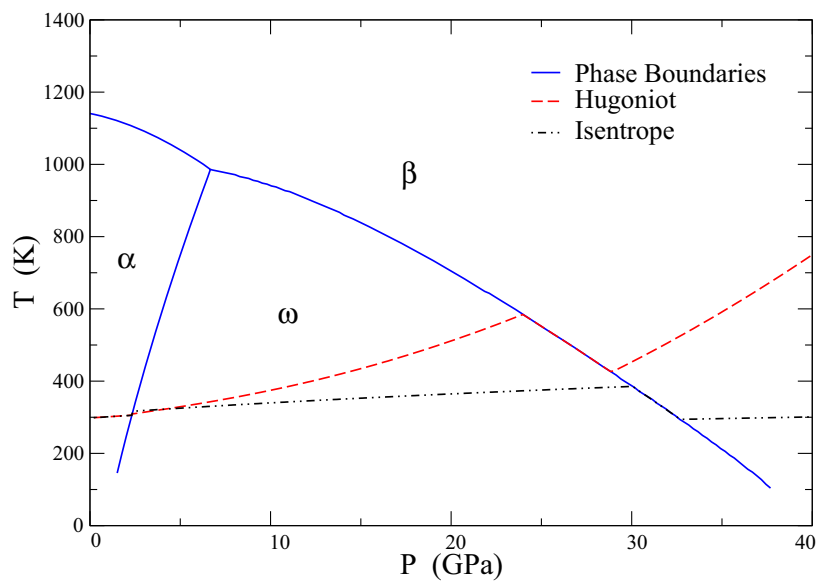

Fig. 2 (color online) Phase diagram of $\mathrm{Zr}$, with the principal Hugoniot and isentrope, according to the EOS described in Ref. [12] 


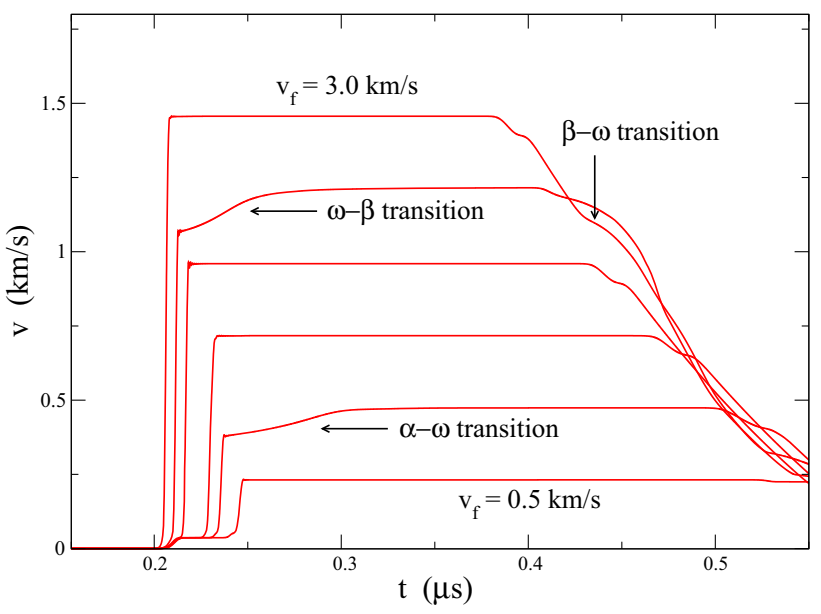

Fig. 3 (color online) Simulated wave profiles for $\mathrm{Al}$ impacting $\mathrm{Zr}$ at varying velocities. The simulated system consists of an Al flyer $1 \mathrm{~mm}$ thick striking a $1 \mathrm{~mm} \mathrm{Zr}$ sample with a $\mathrm{LiF}$ window. The flyer velocity is varied from 0.5 to $3.0 \mathrm{~km} / \mathrm{s}$ in increments of $0.5 \mathrm{~km} / \mathrm{s}$. The velocity at the $\mathrm{Zr} / \mathrm{LiF}$ interface is shown.

give rise to a broad feature in the wave profile. For flyer velocities 1.5 and $2.0 \mathrm{~km} / \mathrm{s}$ there is complete transformation to the $\omega$ phase in the shock front. At $2.5 \mathrm{~km} / \mathrm{s}$, the shock pressure is $30 \mathrm{GPa}$, and the model gives full transformation to the $\omega$ phase in the shock front, followed by complete transformation to the higher pressure $\beta$ phase, which leads to a rounded wave profile. At the highest velocity, the shock pressure is $38 \mathrm{GPa}$, and there is full transformation to the $\beta$ phase in the shock front. In this case there is a structure in the release wave that is attributable to the $\beta$ phase reverting to $\omega$, which is noted in Fig. 3.

Figure 4 shows simulations with the same setup as Fig. 3, except with a narrower range of flyer velocities from 0.6 to $1.6 \mathrm{~km} / \mathrm{s}$ in intervals of $0.2 \mathrm{~km} / \mathrm{s}$. This range focuses on the lower pressure $\alpha-\omega$ transition. This allows us to call attention to some consequences of the transition rate model, Eq. (3). The lowest velocity results in about $6 \%$ transformation to $\omega$ phase, which has hardly any impact on the wave shape. At $v_{f}=0.8 \mathrm{~km} / \mathrm{s}$, the $\omega$ phase fraction increases to 0.65 over $0.4 \mu \mathrm{s}$, resulting a slow increase of the velocity after the shock. This increase is highlighted in the figure by the dashed line at constant velocity of 0.357 $\mathrm{km} / \mathrm{s}$, which is the material velocity at the $\mathrm{Zr} / \mathrm{LiF}$ interface immediately after the shock. For flyer velocities 1.0 and 1.2 $\mathrm{km} / \mathrm{s}$, the $\alpha-\omega$ transition goes to completion, but slowly enough to lead to a gradual rise of the velocity.

In an equilibrium picture of shock-induced phase transitions $[10,24]$, the plastic wave splits into two waves conventionally denoted the $P_{1}$ and $P_{2}$ waves. The $P_{1}$ takes the material up to the phase boundary, and its amplitude and speed are material properties. From Fig. 4, it is seen that the present model departs from this picture. Both the

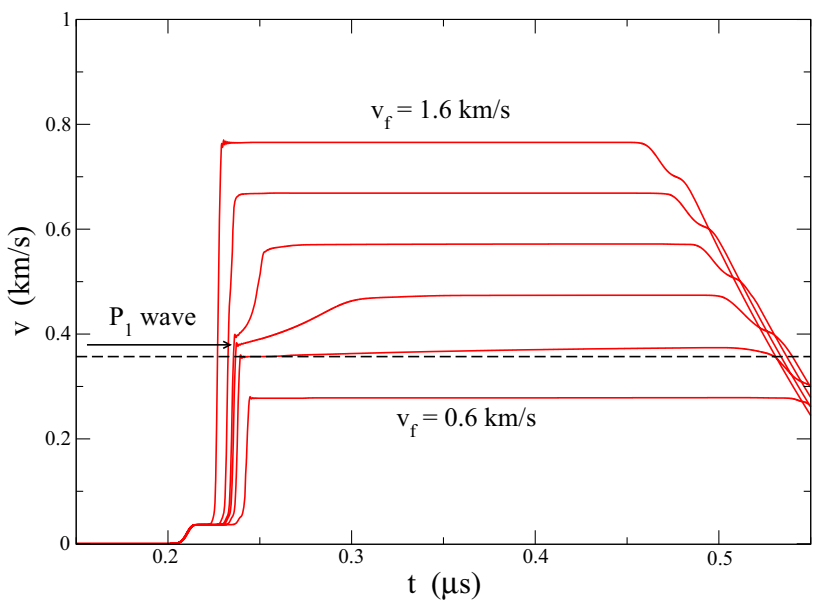

Fig. 4 (color online) Same as Fig. 3, except that the flyer velocity is varied from 0.6 to $1.6 \mathrm{~km} / \mathrm{s}$ in increments of $0.2 \mathrm{~km} / \mathrm{s}$ to focus on the $\alpha-\omega$ transition region. The thin dashed black line is at $v=0.357 \mathrm{~km} / \mathrm{s}$. It is included to draw attention to the slow drift of the velocity after the shock for the case $v_{f}=0.8 \mathrm{~km} / \mathrm{s}$.

amplitude, and the speed, as indicated by the arrival time, increase with increasing shock strength. This phenomenon has been observed in $\mathrm{Ti}[13,20]$ and $\mathrm{Zr}$ [7, 28].

Figure 5 shows the pressure and $\omega$ phase fraction for points in the interior of a $2 \mathrm{~mm}$ thick $\mathrm{Zr}$ sample impacted by $\mathrm{Al}$ at $1.0 \mathrm{~km} / \mathrm{s}$. The points shown, ranging from 0.2 to $1.0 \mathrm{~mm}$ into the sample, are unaffected by wave interactions with the $\mathrm{LiF}$ window over this time. The wave shows substantial non-steadiness over this distance scale in both the pressure and phase fraction. There is an evident $P_{1}$ wave, but its amplitude is decaying with distance, and it occurs at a pressure well above the equilibrium transition pressure of $2.3 \mathrm{GPa}$.

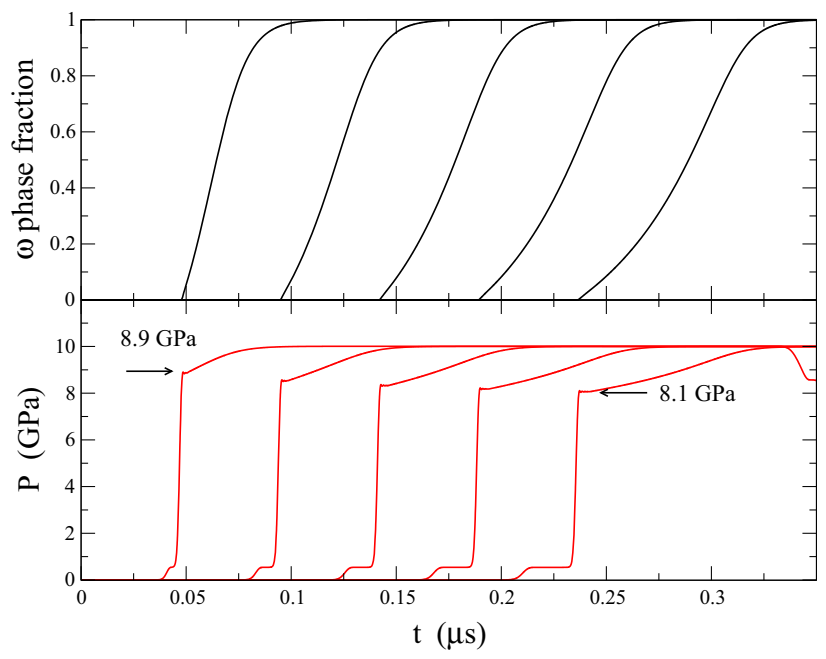

Fig. 5 (color online) In situ pressure and $\omega$ phase fractions for a 2 $\mathrm{mm}$ thick $\mathrm{Zr}$ sample impacted at $1 \mathrm{~km} / \mathrm{s}$ by an $\mathrm{Al}$ flyer. Traces are for Lagrangian positions at 0.2 to $1.0 \mathrm{~mm}$ into the sample from the impact surface. 
The apparent transition pressure, as measured by the $P_{1}$ wave amplitude, lies well above the equilibrium boundary, and depends on non-material factors such as the sample thickness and shock strength. In addition to these dependences, rather small variations in impurity content change the $P_{1}$ wave amplitude substantially $[13,24]$, a phenomenon that is consistent with a reduction in the transition rate by impurities. These factors all contribute to the wide range of transition pressures reported from dynamic experiments, which range from 6.3 to $14 \mathrm{GPa}[21,24,27]$.

Equation (3) for the transformation rate was proposed on purely phenomenological grounds. The discussion of Fig. 1 suggests that there is a limited range of rates that affect the observed wave profile, so a different functional form that behaves the same over this range should give similar results. To test this, Eq. (3) has been replaced with

$R_{i j}=\theta\left(G_{i}-G_{j}\right) \xi_{i j} \sinh \left[\left(G_{i}-G_{j}\right) / C_{i j}\right]$

with the parameters related to those of Eq. (3) by

$C_{i j}=0.2023 B_{i j}$

$\xi_{i j}=0.01069 v_{i j}$.

These coefficients were obtained by least squares fit of the curve in Fig. 1. Repeating the simulations of Figs. 3 and 4 with this model results in curves that are nearly indistinguishable from those obtained with Eq. (3). This confirms that the particular functional form for $R_{i j}$ is not unique, and that other functions with similar behavior would serve as well.

Anderson et al. performed experiments [1] on the $\beta-\gamma$ transition in tin, and obtained similar wave profiles to those shown in Fig. 4. Cox and Robinson [9] obtained good results simulating these experiments using Eq. (3), suggesting usefulness beyond the original applications to $\mathrm{Ti}$ and $\mathrm{Zr}$. They also obtained results of similar quality with linearly increasing rate above a threshold, reinforcing the above observations about the non-uniqueness of the functional form for $R_{i j}$. It would be preferable for the functional form of $R_{i j}$ to reflect the physics of the underlying process, so that meaning could be attributed to the parameters, and the values of the parameters could be estimated based on other physical properties.

\section{Growth Model}

This section illustrates the possibility of extending the model to incorporate micro-scale processes. A particular case is considered, where the transformation rate is limited by growth, and there is a fixed density $n$ of nucleation sites. These assumptions may be realistic for polycrystalline materials where, for instance, grain-scale fluctuations of the stress or temperature may initiate transformations. However, the main motivation for making them here is that they allow for a closed model without additional dynamical state variables.

Rather than the transformation rate $R$ itself, it is assumed that the phase interface velocity $v$ is a function of the thermodynamic driving force. The assumption of a fixed density of nucleation sites allows for the radius of the second phase domains (here assumed to be spherical) to be written $r=(3 \lambda / 4 \pi n)^{1 / 3}$, allowing us to write,

$\dot{\lambda}_{2}=(36 \pi n)^{1 / 3} \lambda_{2}^{2 / 3} v$.

(The velocity $v$ and density $n$ are taken in the Lagrangian frame). Equation (16) is valid when $\lambda_{2}$ is small so that the phase 2 domains do not impinge on each other. A geometric factor $1-\lambda_{2}$ is usually included [3] to compensate for this impingement. We have seen in the discussion following Eq. (1) that this factor is already included in the master equation. Thus, Eq. (3) is replaced by

$R_{i j}=(36 \pi n)^{1 / 3} \lambda_{j}^{2 / 3} v_{i j}\left(G_{i}-G_{j}\right)$,

where $v_{i j}$ is the interface velocity of phase $j$ growing into phase $i$. In contrast to Eqs. (3) and (17) brings in an explicit dependence of $R_{i j}$ on $\lambda_{j}$, which reflects the surface area of the $j$ phase domains.

Consider a situation in which initially $\lambda_{2}=0, \lambda_{1}=1$, and the thermodynamic state is suddenly switched to one where phase 2 is stable. Equation (17) gives $\lambda_{2} \propto t^{3}$ at short time, whereas the model of Eq. (3) gives $\lambda_{2} \propto t$. The $t^{3}$ dependence arises from the $\lambda_{j}^{2 / 3}$ factor. It is considered indicative of isotropic growth, as has been assumed here. In both cases, the second phase fraction approaches one at large times. The sigmoidal curve curve from Eq. (17) is generally thought to be more realistic [3, 8]. This is illustrated in Fig. 6, which shows the time evolution of the second phase fraction according to the Andrews model [2], the present model using Eqs. 2 and 3, and the growth model using Eqs. 2 and 17. The time scale is set by different variables in the different models. The graph uses a dimensionless time variable to show the models together. For the Andrews model, it is $L\left(G_{1}-G_{2}\right) t$, where $L$ is a constant. For the present model it is $R_{12}\left(G_{1}-G_{2}\right) t$, with $R_{12}$ given by Eq. (3). For the growth model, the time variable is $(36 \pi n)^{1 / 3} v_{12}$. Use of Eq. (17) requires that $\lambda_{2}$ be initialized to a finite value in order to avoid the solution $\lambda_{2}=0$. A preferable option would be to devise a a physical criterion for the formation of critical nuclei.

The exponential dependence of $R_{i j}$ on the thermodynamic driving force as in Eq. (3) or Eq. (14) is an important feature of the present model, and is behind many of the qualitative behaviors shown in Sect. 4. In order for the 


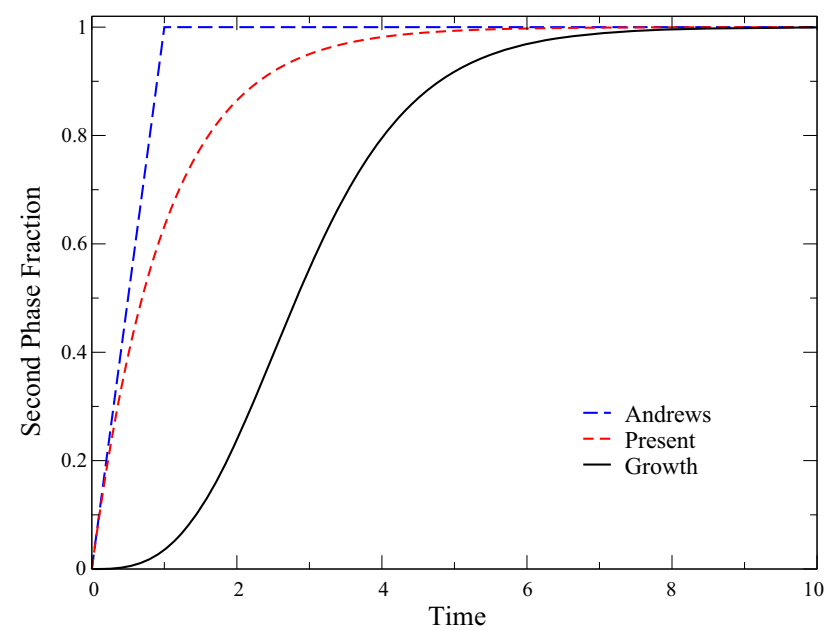

Fig. 6 (color online) Time evolution of the second phase fraction in various models. Initially the second phase fraction is zero, and the thermodynamic state is switched to one in which phase 2 is stable at $t=0$. The blue long-dashed curve is the model of Andrews [2], the red short-dashed curve is the present model of Eqs. (2) and (3). The solid black curve is the growth model of Eqs. (2) and (17). The time units are different for the various models, as described in the text.

growth model, Eq. (17) to replicate this, the phase interface velocity $v_{i j}$ would have to have an exponential dependence on $G_{i}-G_{j}$. Becker [4] described an activated model for the phase interface velocity for the $\beta \rightarrow \alpha$ phase transition in Sn. In their model, the growth rate reaches a finite asymptotic value as the driving force increases with a fixed activation barrier, rather than increasing without limit, as the present model does. Singh et al. [25] reported evidence (with large uncertainties) of a decrease in the activation energy for the $\alpha-\omega$ transition in $\mathrm{Ti}$ with increasing pressure. Such a decrease would lead to an exponential growth of the transition rate with pressure, in qualitative agreement with the present phenomenological model.

\section{Conclusions}

A model has been described for phase transitions under dynamic compression that operates at the macro scale, where second phase domains are not resolved. The time evolution of the phase fractions obeys the master equation, so that the normalization is preserved, and complete transformation is approached asymptotically. The rate is taken to depend on the thermodynamic driving force exponentially, rather than linearly as has been more typical. This exponential dependence gives rise to some interesting phenomena, such as decay of the P1 wave. Some of these are illustrated by the calculations in Sect. 4 .

The model has two parameters for each pair of phases, which must be determined empirically from wave profile data. It would be preferable for the model to be based on a mechanistic picture so that the parameters can be obtained from independent data or calculations. In Sect. 5, I have shown an example of a mechanistically based variant of the model, in which domain growth is the rate-limiting process. In this version, the phase interface velocity must be specified as a function of the thermodynamic driving force, as opposed to the net transformation rate. It may be possible to evaluate this function via molecular dynamics simulations [17]. Experimental input will be required to confirm any micro-scale mechanism. The $\alpha-\omega$ transition in $\mathrm{Ti}$ and $\mathrm{Zr}$ has the advantageous feature that the high pressure phase is retained on release to ambient pressure, allowing microstructural investigation of shocked samples after the fact [6]. Recent developments in light sources are starting to allow X-ray diffraction and imaging at the 1-2 $\mu \mathrm{m}$ length scale on dynamic compression time scales [11, 19], opening the possibility of in situ examination of microstructural evolution.

Acknowledgments The author gratefully acknowledges support from Advanced Simulation and Computing, and Science Campaign 1 at LANL, which is operated by LANS, LLC for the NNSA of the U.S. DOE under Contract No. DE-AC52-06NA25396.

\section{References}

1. Anderson WW, Cverna F, Hixson RS, Vorthman J, Wilke MD, Gray GT, Brown KL (2000) Phase transition and spall behavior in beta-tin. AIP Conf Proc 505:443-446

2. Andrews DJ (1971) Calculation of mixed phases in continuum mechanics. J Comput Phys 7(2):310-326. doi:10.1016/00219991(71)90092-1

3. Avrami M (1940) Kinetics of phase change. II transformationtime relations for random distribution of nuclei. J Chem Phys 8(2):212-224. doi:10.1063/1.1750631

4. Becker J (1958) On the quality of gray tin crystals and their rate of growth. J Appl Phys 29(7):1110-1121

5. Boettger JC, Wallace DC (1997) Metastability and dynamics of the shock-induced phase transition in iron. Phys Rev B 55(5):2840-2849. doi:10.1103/PhysRevB.55.2840

6. Brown DW, Almer JD, Balogh L, Cerreta EK, Clausen B, Escobedo-Diaz JP, Sisneros TA, Mosbrucker PL, Tulk EF, Vogel SC (2014) Stability of the two-phase (alpha/omega) microstructure of shocked zirconium. Acta Mater 67:383-394. doi:10.1016/ j.actamat.2013.12.002

7. Cerreta EK, Addessio FL, Bronkhorst CA, Brown DW, Escobedo JP, Fensin JP, Gray GT III, Lookman T, Rigg PA, Trujillo CP (2014) he influence of peak shock stress on the high pressure phase transformation in Zr. J Phys Conf Ser 500:032003. doi:10. 1088/1742-6596/500/3/032003

8. Christian JW (1965) The Theory of Transformations in Metals and Alloys. Pergamon, Oxford

9. Cox GA, Robinson CM (2009) Empirical multi-phase EOS modelling issues. AIP Conf Proc 1195:1195-1200

10. Duval GE, Graham RA (1977) Phase-transitions under shockwave loading. Rev Mod Phys 49(3):523-579. doi:10.1103/ RevModPhys.49.523

11. Gleason AE, Bolme CA, Lee HJ, Nagler B, Galtier E, Milathianaki D, Hawreliak J, Kraus RG, Eggert JH, Fratanduono DE, 
Collins GW, Sandberg R, Yang W, Mao WL (2015) Ultrafast visualization of crystallization and grain growth in shock-compressed sio2. Nat Commun 6:8191. doi:10.1038/ncomms9191

12. Greeff CW (2005) Phase changes and the equation of state of $\mathrm{Zr}$. Model Simul Mater Sci Eng 13(7):1015-1027. doi:10.1088/09650393/13/7/001

13. Greeff CW, Rigg PA, Knudson MD, Hixson RS, Gray GT (2004) Modeling dynamic phase transitions in Ti and Zr. AIP Conf Proc 706:209-212

14. Greeff CW, Trinkle DR, Albers RC (2001) Shock-induced alphaomega transition in titanium. J Appl Phys 90(5):2221-2226. doi:10.1063/1.1389334

15. Greeff CW, Trinkle DR, Albers RC (2002) Alpha-omega transition in Ti: equation of state and kinetics. AIP Conf Proc 620(1):225-228. doi:10.1063/1.1483521

16. Hayes DB (1975) Wave-propagation in a condensed medium with $\mathrm{N}$ transforming phases-application to solid-I-solid-II-liquid bismuth. J Appl Phys 46(8):3438-3443. doi:10.1063/1. 322065

17. Hennig RG, Lenosky TJ, Trinkle DR, Rudin SP, Wilkins JW (2008) Classical potential describes martensitic phase transformations between the alpha, beta, and omega titanium phases. Phys Rev B 78(5):054121. doi:10.1103/PhysRevB.78.054121

18. Jacobsen MK, Velisavljevic N, Sinogeikin SV (2015) Pressureinduced kinetics of the alpha to omega transition in zirconium. J Appl Phys 118(2):025902. doi:10.1063/1.4926724

19. Jensen BJ, Ramos KJ, Iverson AJ, Bernier J, Carlson CA, Yeager JD, Fezzaa K (2014) Dynamic experiment using IMPULSE at the advanced photon source. J Phys Conf Ser 500:042001. doi:10. 1088/1742-6596/500/4/042001
20. Kiselev AN, Falkov AA (1982) Phase-transformation in titanium in shock-waves. Combust Explos Shock Waves 18(1):94-97. doi:10.1007/BF00783939

21. Kutsar AR, Pavlovskii MN, Kamissarov VV (1984) Observation of a 2-wave shock configuration in zirconium. JETP Lett 39(9):480-483

22. Mabire C, Hereil PL (2000) Shock induced polymorphic transition and melting of tin. AIP Conf Proc 505:93-96

23. Reichl LE (1980) A Modern Course in Statistical Physics. University of Texas Press, Austin

24. Rigg PA, Greeff CW, Knudson MD, Gray GT, Hixson RS (2009) Influence of impurities on the alpha to omega phase transition in zirconium under dynamic loading conditions. J Appl Phys 106(12):123532. doi:10.1063/1.3267325

25. Singh A, Mohan M, Divakar C (1983) Pressure-induced alphaomega transformation in titanium: Features of the kinetics data. J Appl Phys 54(10):5721-5726

26. Singh AK, Mohan M, Divakar C (1982) The kinetics of pressureinduced alpha-omega transformation in Ti. J Appl Phys 53(2):1221-1223. doi:10.1063/1.330530

27. Song SG, Gray GT (1995) Microscopic and crystallographic aspects of retained omega-phase in shock-loaded zirconium and its formation mechanism. Philos Mag A 71(2):275-290

28. Ying-Hua L, Lin Z, Ling-Cang C (2007) Effects of shock pressure on transition pressure in Zr. Chin Phys Lett 24(3):784-785

29. Zhang J, Zhao Y, Pantea C, Qian J, Daemen LL, Rigg PA, Hixson RS, Greeff CW III, Gray GT, Yang Y, Wang L, Wang Y, Uchida $\mathrm{T}$ (2005) Experimental constraints on the phase diagram of elemental zirconium. J Phys Chem Solids 66(7):1213-1219. doi:10. 1016/j.jpcs.2005.03.004 